\title{
Addendum to Paper Entitled "Do Prime Numbers Obey a Three Dimensional Double Helix?"
}

\author{
Peter Bissonnet \\ Independent and Unaffiliated Researcher, Fusagasuga, Cundinamarca, Colombia, South America \\ Email: peterkey1624@gmail.com
}

How to cite this paper: Bissonnet, $P$. (2017) Addendum to Paper Entitled "Do Prime Numbers Obey a Three Dimensional Double Helix?" Journal of Applied Mathematics and Physics, 5, 1825-1836. https://doi.org/10.4236/jamp.2017.59154

Received: July 31, 2017

Accepted: September 24, 2017

Published: September 27, 2017

Copyright $\odot 2017$ by author and Scientific Research Publishing Inc. This work is licensed under the Creative Commons Attribution International License (CC BY 4.0).

http://creativecommons.org/licenses/by/4.0/

\section{(c) (i) Open Access}

\begin{abstract}
This paper again specifies the major points of the article "Do Prime Numbers Obey a Three-Dimensional Double Helix?" [1] which was received on February 16, 2006 by Hadronic Journal. New information has been added and elucidated upon, such as why the numbers 2 and 3 are not considered true prime numbers, and why $s$ in the following formulas for $6 s-1$ and for $6 s+1$ is really a composite number equal to the sum of two other numbers, suggesting that $s$ is always to be considered as an integer. Other new information is added as well, such as how an engineer in a matter of seconds decomposed a large prime product into its constituent primes using basic software and won a contract for his firm.
\end{abstract}

\section{Keywords}

Prime Numbers, Prime Products, Number Theory

\section{Introduction}

This addendum clears the air once and for all by reaffirming that the above author's paper of 2006 provides the first proof or initial discovery of the double helices of prime numbers, which, unfortunately, also includes prime products, by using the seven-column array. This discovery was made by letting the sequential set of integers found in a seven-column array with an infinite number of rows to "speak for themselves". When my paper of 2006 stated that 2 and 3 were not prime numbers, many mathematicians, instead of listening to what mathematics was telling them, had to "save" the definition of prime numbers at all cost by throwing a "life preserver" to the numbers 2 and 3, because these numbers could not swim in the sea of true prime numbers. The life preserver was in the form of 
double helices of their invention which allowed 2 and 3 to fall along these helices. Incidentally, where did these mathematicians obtain these double helices from anyway? The author proved his assertion of the double helices. There must be hundreds of thousands of curves of all types in mathematics, including both two and three-dimensional curves. How did these mathematicians happen to choose the double helices...why not single helices or triple helices? With such Fermat-type perspicacity, such "intellects" should easily be able to solve the Riemann Hypothesis! Do these mathematicians actually think that the double helices derived from the aforementioned seven column array were actually some sort of deception by mathematics? Do they think that mathematics can lie? Do they think that this seven-column array represents some sort of inconsistency in the foundations of mathematics? Not listening to what mathematics is saying only does a disservice to mathematics and for what reason? The saving of nationalistic and historic mathematical pride? The gung-ho desire for fame, fortune, and recognition? To put it bluntly, these theories that include 2 and 3 as part of the double helix system are erroneous and smack of finagling in order to force fit the numbers 2 and 3 into the prime number and prime product structure at any cost. The symbolic prime number scheme discovered by the author, namely, $\mathrm{H}_{1}$ $\otimes \mathrm{H}_{1}=\mathrm{H}_{2}=\mathrm{H}_{2} \otimes \mathrm{H}_{2}$ and $\mathrm{H}_{1} \otimes \mathrm{H}_{2}=\mathrm{H}_{1}$ shows that the product of prime numbers and even products of prime numbers with other prime products form a closed system. Including 2 and 3 into the set of prime numbers results in a system that is no longer closed. It's like multiplying two vectors together: one no longer ends up with a vector in the set of vectors; one ends up with a quaternion.

\section{Why Does the Number 42 Show up in Prime Numbers?}

The author believes that he read this in a book from years ago, possible entitled Mathematical Mountaintops by John Casti. The author has not used this as a reference, because he does not remember for sure if that is where he read it. The author's theory explains the repetition of 42 that occurs in prime numbers, as well as the number 14, that shows up occasionally. The equations representing the two-dimensional representation of the prime number double helix make this very clear.

\section{Case I: Difference of two primes on Helix 1}

The equation for the two-dimensional representation of Helix 1 is (remembering that the x's represent the column values and the n's represent the values of the complex (or grouping) in which the prime number is located).

$$
P_{1}\left(n_{1}\right) x_{1}=6 x_{1}-35-42 n_{1}
$$

but remembering that $P_{1}$ is negative, we take the absolute value as

$$
\left|P_{1}\left(n_{1}\right) x_{1}\right|_{A}=-6 x_{1}+35+42 n_{1}
$$

as representing prime number $A$ on Helix 1. Similarly, for prime number $B$ on Helix 1 which is greater than $A$, we have

$$
\left|P_{1}\left(n_{2}\right) x_{2}\right|_{B}=-6 x_{2}+35+42 n_{2}
$$




$$
\begin{aligned}
\left|P_{1}\left(n_{2}\right) x_{2}\right|_{B}-\left|P_{1}\left(n_{1}\right) x_{1}\right|_{A} & =-6 x_{2}+35+42 n_{2}-\left(-6 x_{1}+35+42 n_{1}\right) \\
& =-6 x_{2}+35+42 n_{2}+6 x_{1}-35-42 n_{1} \\
& =6\left(x_{1}-x_{2}\right)+42\left(n_{2}-n_{1}\right)
\end{aligned}
$$

Thus, we can see that there is an integral multiple of 42 between two prime numbers on Helix 1 if and only if $x_{1}-x_{2}=0$.

Example (1) $x=2$ and prime number $A$ is 23 , and $B$ is 107 , we have $n_{2}-n_{1}=2$

Example (2) $x=2$ and $A$ is 23 and $B$ is 233 , we have $n_{2}-n_{1}=5$.

Example (3) $x=5$ and $A$ is 5 and $B$ is 47 , we have $n_{2}-n_{1}=1$.

\section{Case II: Difference of two primes on Helix 2}

The equation for the two-dimensional representation of Helix 2 is

$$
P_{2}\left(n_{1}\right) x_{1}=6 x_{1}-49-42 n_{1}
$$

and taking the absolute value, we obtain

$$
\left|P_{2}\left(n_{1}\right) x_{1}\right|_{A}=-6 x_{1}+49+42 n_{1}
$$

represents prime number $A$ on Helix 2

$$
\left|P_{2}\left(n_{2}\right) x_{2}\right|_{B}=-6 x_{2}+49+42 n_{2}
$$

represents prime number $B$ on Helix 2 which is greater than $A$

$$
\begin{aligned}
\left|P_{2}\left(n_{2}\right) x_{2}\right|_{B}-\left|P_{2}\left(n_{1}\right) x_{1}\right|_{A} & =-6 x_{2}+49+42 n_{2}-\left(-6 x_{1}+49+42 n_{1}\right) \\
& =-6 x_{2}+49+42 n_{2}+6 x_{1}-49-42 n_{1} \\
& =6\left(x_{1}-x_{2}\right)+42\left(n_{2}-n_{1}\right)
\end{aligned}
$$

Thus, we can see that there is an integral multiple of 42 between two prime numbers on Helix 2 if and only if $x_{1}-x_{2}=0$.

Example (1) $x=1$ and prime number $A$ is 43 and $B$ is 127, we have $n_{2}-n_{1}=2$

Example (2) $x=1$ and prime number $A$ is 43 and $B$ is 211, we have $n_{2}-n_{1}=4$

Example (3) $X=4$ and prime number $A$ is 67 and $B$ is 151, we have $n_{2}-n_{1}=2$

Case III: Difference of two primes - one on Helix 1 and the other on Helix 2

$$
\left|P_{1}\left(n_{A}\right) x_{A}\right|_{A}=-6 x_{A}+35+42 n_{A}
$$

as representing prime number $A$ on Helix 1.

$$
\left|P_{2}\left(n_{B}\right) x_{B}\right|_{B}=-6 x_{B}+49+42 n_{B}
$$

represents prime number $B$ on Helix 2 which is assumed to be greater than $A$. If the situation were reversed, it would simply be a matter of sign difference.

$$
\begin{aligned}
\left|P_{2}\left(n_{B}\right) x_{B}\right|_{B}-\left|P_{1}\left(n_{A}\right) x_{A}\right|_{A} & =-6 x_{B}+49+42 n_{B}-\left(-6 x_{A}+35+42 n_{A}\right) \\
& =-6 x_{B}+49+42 n_{B}+6 x_{A}-35-42 n_{A} \\
& =6\left(x_{A}-x_{B}\right)+14+42\left(n_{B}-n_{A}\right)
\end{aligned}
$$

Thus, we can have a difference of multiples of 42 if and only if $6\left(x_{A}-x_{B}\right)+14=0$ or $x_{B}-x_{A}=7 / 3$, but this is impossible since the $\mathrm{x}$ 's are integers. Therefore, we must draw the conclusion that in no case can there be dif- 
ferences between prime numbers of multiples of 42 , if the prime numbers reside on different helices.

\section{The $s$ in $6 s-1$ and $6 s+1$ is Actually a Composite Number}

$6 s-1$ and $6 s+1$, where $s \geq 1$, are used in designating the terms, respectively, of Helix 1 beginning with prime number 5 and Helix 2 beginning with prime number 7. Few people realize that the $s$ values are themselves composite numbers which are sums of two other numbers. Please note that Helices 1 and 2 include only columns 1-6, while column 7 only includes prime products involving 7 , with the lone exception of prime number 7 on Helix 2 which is in row 1, column 7 (see Table 2).

The first set of double parallel lines in Table 2 is $n=0$ or complex 0 , the second set of double parallel lines is $n=1$ or complex 1 , and so on. The breakdown of $s$ is as follows:

$s=r+n$, where $r$ is the row number of where the prime number is located and $n$ is the complex it is located in.

Prime numbers or prime products falling on $\mathrm{H} 1$ are denoted ( $x=1$ to 6 ) by

$$
P_{1}(n) x=6 x-35-42 n
$$

where $x$ represents the column number and $\mathrm{n}$ represents the complex number. For H2 (prime number 7 is included on $\mathrm{H} 2$ by including $x=7$ only for row $r=1$, see Table 2 for a visual representation) in similar fashion,

$$
P_{2}(n) x=6 x-49-42 n
$$

It is also true that the numbers along the helical lines can be represented by

$$
P_{r, x}=7(r-1)+x
$$

again, where $r$ is the row number and $x$ is the column number. Solving for $x$ and substituting in the above two equations, we obtain

$$
\begin{aligned}
P_{1}(n) x=6\left[P_{r, x}-7(r-1)\right]-35-42 n & =6 P_{r, x}-42(r-1)-35-42 n \\
& =6 P_{r, x}-42(r+n)+7
\end{aligned}
$$

We now note that $P_{1}(n) x$ is always negative and $P_{r, x}$ is always positive, so we let $P_{1}(n) x=-P_{r, x}$.

$$
-P_{r, x}=6 P_{r, x}-42(r+n)+7
$$

which rearranges into

$$
P_{r, x}=6(r+n)-1=6 s-1
$$

Similarly, for $\mathrm{H} 2$, we have

$$
P_{2}(n) x=6 x-49-42 n=6\left[P_{r, x}-7(r-1)\right]-49-42 n
$$

Again, we let

$$
\begin{array}{r}
P_{2}(n) x=-P_{r, x} \text { and upon rearranging, we obtain } \\
P_{r, x}=6(r+n)+1=6 s+1
\end{array}
$$


Table 1. Values of $s=r+n$.

\begin{tabular}{cccc}
\hline Prime number & $s$ & $r$ & $n$ \\
\hline $293(\mathrm{H} 1)$ & 49 & 42 & 7 \\
$197(\mathrm{H} 1)$ & 33 & 29 & 4 \\
$181(\mathrm{H} 2)$ & 30 & 26 & 4 \\
$241(\mathrm{H} 2)$ & 40 & 35 & 5 \\
$97(\mathrm{H} 2)$ & 16 & 14 & 2 \\
$239(\mathrm{H} 1)$ & 40 & 35 & 5 \\
$199(\mathrm{H} 2)$ & 33 & 29 & 4 \\
\hline
\end{tabular}

which concludes the proof. See Table 1 for actual examples.

\section{Why are the Numbers 2 and 3 not True Prime Numbers?}

From the point of view of this paper, the numbers 2 and 3 are not prime numbers, even though they fall under the definition of prime numbers. This paper shows quite clearly, that the true prime numbers begin with 5 and 7 . This paper takes the position that all true primes fall along the helical curves designated as $\mathrm{H} 1(6 s-1)$ and $\mathrm{H} 2(6 s+1)$, where $s \geq 1$. Further, products of true primes also fall along one or the other of $\mathrm{H} 1$ or $\mathrm{H} 2$, with the exception of those involving 7 , excepting column 7 row 1 . This section will therefore deal with the larger sets of $6 s-1$ and $6 s+1(x=1$ to 7$)$, which includes $\mathrm{H} 1$ and $\mathrm{H} 2(x=1$ to 6$)$. If we consider the product of primes, then symbolically

$$
\mathrm{H}_{1} \otimes \mathrm{H}_{1}=\mathrm{H}_{2}=\mathrm{H}_{2} \otimes \mathrm{H}_{2} \text { and } \mathrm{H}_{1} \otimes \mathrm{H}_{2}=\mathrm{H}_{1}
$$

The Comparison of 2 and 3 with the Prime Numbers Beginning with 5 on Helix 1 and 7 on Helix 2 Results in the Following

Characteristic 1: Obeys the classical definition of prime numbers?

\begin{tabular}{llllllllllll}
\hline & 2 & 3 & 5 & 7 & 11 & 13 & 17 & 19 & 23 & 31 & Etc. \\
\hline Yes & $\sqrt{ }$ & $\sqrt{ }$ & $\sqrt{ }$ & $\sqrt{ }$ & $\sqrt{ }$ & $\sqrt{ }$ & $\sqrt{ }$ & $\sqrt{ }$ & $\sqrt{ }$ & $\sqrt{ }$ & \\
No & & & & & & & & & & & \\
\hline
\end{tabular}

Characteristic 2: Falls along either one or the other of $6 s-1$ or $6 s+1$, where $s$ $\geq 1$ ?

\begin{tabular}{llllllllllll}
\hline & 2 & 3 & 5 & 7 & 11 & 13 & 17 & 19 & 23 & 31 & Etc. \\
\hline Yes & & & $\sqrt{ }$ & $\sqrt{ }$ & $\sqrt{ }$ & $\sqrt{ }$ & $\sqrt{ }$ & $\sqrt{ }$ & $\sqrt{ }$ & $\sqrt{ }$ & \\
No & $\sqrt{ }$ & $\sqrt{ }$ & & & & & & & & & \\
\hline
\end{tabular}

Characteristic 3: Do products of prime numbers fall along $6 s-1$ or $6 s+1$, where $s \geq 1$ ?

\begin{tabular}{|c|c|c|c|c|c|c|c|c|c|c|c|}
\hline & $2 \times 2$ & $3 \times 3$ & $2 \times 3$ & $2 \times 5$ & $3 \times 5$ & $2 \times 11$ & $3 \times 11$ & $2 \times 7$ & $3 \times 7$ & $2 \times 13$ & $3 \times 13$ \\
\hline Yes & & & & & & & & & & & \\
\hline No & $\sqrt{ }$ & $\sqrt{ }$ & $\sqrt{ }$ & $\sqrt{ }$ & $\sqrt{ }$ & $\sqrt{ }$ & $\sqrt{ }$ & $\sqrt{ }$ & $\sqrt{ }$ & $\sqrt{ }$ & $\sqrt{ }$ \\
\hline
\end{tabular}




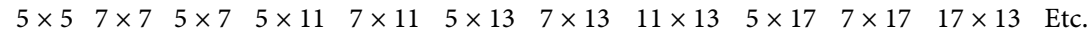

\begin{tabular}{llllllllllll}
\hline Yes & $\sqrt{H 2}$ & $\sqrt{ }$ & $\sqrt{ }$ & $\sqrt{\mathrm{H} 1}$ & $\sqrt{ }$ & $\sqrt{\mathrm{H} 1}$ & $\sqrt{ }$ & $\sqrt{\mathrm{H} 1}$ & $\sqrt{\mathrm{H} 2}$ & $\sqrt{ }$ & $\sqrt{\mathrm{H} 1}$
\end{tabular}

No

Thus, even though 2 and 3 satisfy the classical definition of prime numbers, neither they nor their products fall along the double helices or the larger set of $6 \mathrm{~s}$ -1 or $6 s+1$. Whenever one tries to form a group or set of objects, one chooses those items which possess all of the characteristics of the group. Clearly, based upon the above simplistic analysis, 2 and 3 only satisfy 1 of the 3 common characteristics of prime numbers and that is the reason that this paper cannot consider 2 or 3 to be true prime numbers. Perhaps they form a lower grade set of prime numbers. True prime numbers obey $(6 m+a)(6 n+b)=(6 r+c)$, where $m, n$, and $r$ are integers greater than or equal to 1 and $a, b$, and $c$ are +1 or -1 .

\section{Discoverable Mathematics Instead of Postulate Driven Mathematics?}

The Columbia Encyclopedia, Columbia University Press, Sixth Edition, (2000) defines mathematics: the deductive study of numbers, geometry, and various abstract constructs, or structures; the latter often "abstract" the features common to several models derived from the empirical, or applied sciences, although many emerge from purely mathematical or logical considerations.

The author did not use deduction, logical propositions, postulates, or axioms, in the sense of the above definition, in arriving at the above 7 column array. Neither did he have to resort to large and complex computer programs to discover the prime number double helices nor did he plagiarize other people's works. In fact, the author used methods more in line with exploratory physics in arriving at the above double helices. The author believes that there may be areas of mathematics which fall more into the category of being discoverable, as opposed to being postulate or axiom driven, from which the deductive inferences in the philosophy of logic known as Modus Ponens and Modus Tollens, along with the four syllogistic figures and the nineteen valid moods can be invoked to derive other true statements.

If what the author suspects is true, namely, that these double helices are in the discoverable category of mathematics (i.e. overlapping with experimental physics), then it may be a fair assertion that nature has a hand in determining which prime numbers are to be considered true prime numbers, irrespective of man's preconceived definitions of what is or is not a prime number. See the following Table 2 for an example of the prime number helices.

A bit of history is necessary in understanding Table 2, which was created by taking seven columns of cells in an Excel spreadsheet and filling in the cells from left to right starting from 1 to 7 on the first row, 8 to 14 on the second row, 15 to 21 on the third row, etc. The next step was to blank out all of the non-prime numbers, which even included products of prime numbers. What was left is 
Table 2. Example of the Prime Number Double Helices.

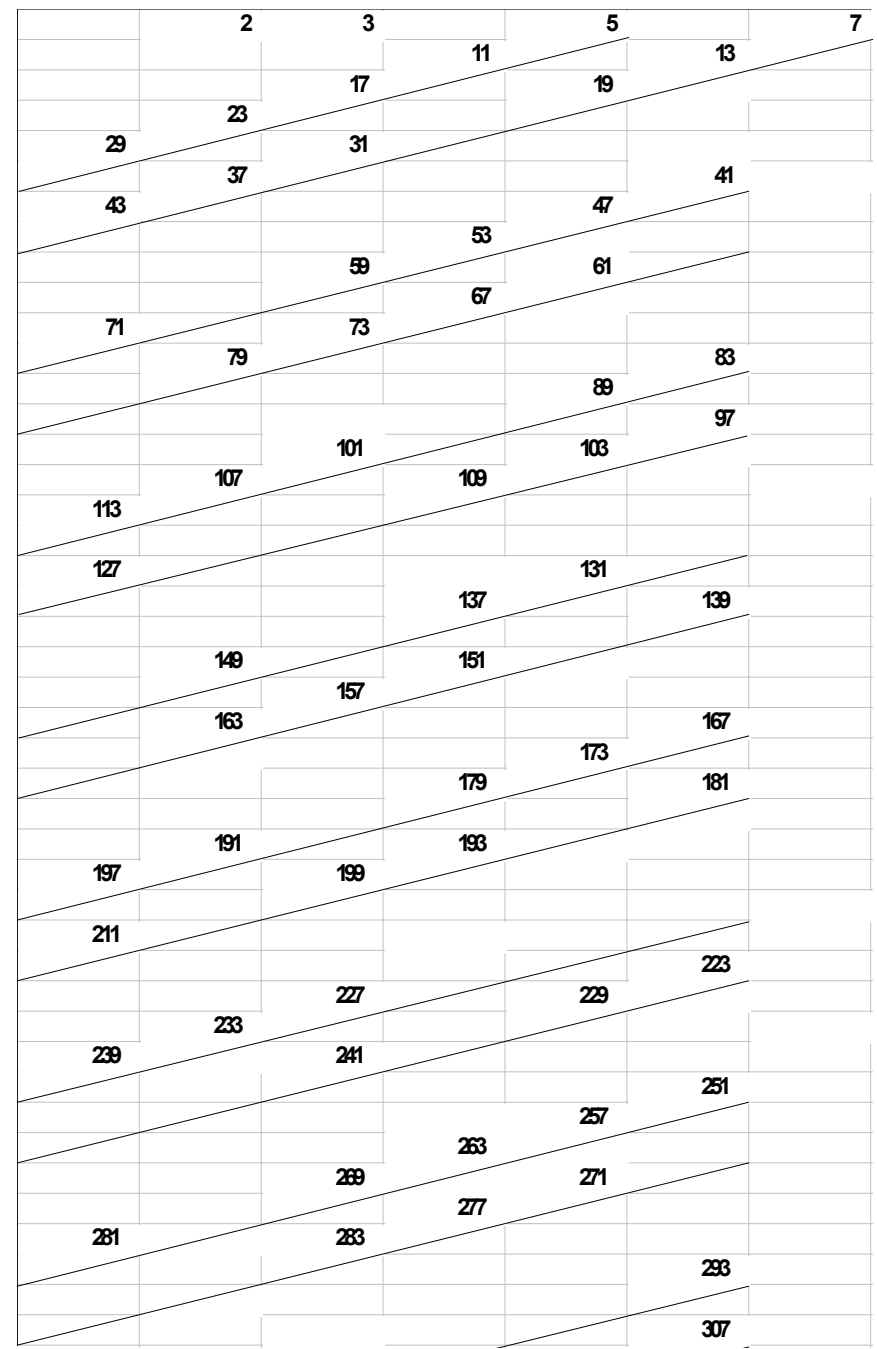

what the reader sees above. Noticing these parallel lines on the computer screen, the author drew in straight lines and printed out the page and left to get a cup of coffee. This was done one Sunday morning, while still in pajamas, back in December 2005. During the interim, the author's wife cut around the lines and eliminated the $7^{\text {th }}$ column (except for row 1), as it was blank, containing nothing but products of 7 . She folded the paper around in a cylinder and noticed that the lines met in the back. She called my attention to it, and we noticed that the lines ran around the cylinder of paper in the form of a double helix (yes, the author knows what some of you are thinking; the author asked his wife, but she declined to have her name on the original 2006 paper). Again, this was a discovery not a deductive process, and, therefore, lies outside of the definition of mathematics, which emphasizes postulate/deductive driven processes. It also speaks to those philosophers who advocate that mathematics, as a result of being postulate driven or concerned with ideas originating in the mind of mathematicians, does not exist in the real world but exists only in the mind of man or mathematicians. The author believes that this viewpoint is absurd in its totality. Let us look at the 
first line, as we progress from 5 to 11 to 17 to 23 to 29 , we see that the difference is 6. Looking at the second line, we progress from 7 to 13 to 19 , we notice that there is a blank where 25 should be, then proceeding to 31,37 and 43 . The number 25 is the first of the prime products being equal to $5 \times 5$. If we look at the group or complex $n=6$, which is the last set of complete double lines, we start at 251 to 257 to 263 to 269 to 275 , which is missing, and on to 281. The number 275 is a product of three prime numbers $275=5 \times 5 \times 11$. As prime numbers increase in value towards infinity, what might be considered as the prime number density decreases [3] [4]. This is due to the fact that the prime numbers are shoved further and further apart by the intercession of prime products and of multiple prime products. Prime numbers appear random, but they are not [2]. Twin or paired primes noted in the 2006 paper must also be infinite in number [3].

\section{The Runway Problem-How an Engineer Dealt in a Practical and Simple Way with a Large Prime Product}

This paper relates simply to an imaginary off-hand conversation which the author heard about, but the numbers seem real. The conversation related to an un-named procurement official at some level of government who, along with other procurement officers in some un-named defense department of some un-named country, wanted a large runway built for experimental aircraft across a very large dry lake bed. These procurement personnel planned on having the project built by some un-named government contractor; however, this chosen government contractor would not be selected by the usual bid mechanism from a list of potential government contractors. Instead, they would choose the winning contractor by giving their engineers a bit of a mental exercise, because they wanted the most competent government contractor, irrespective of low bid, medium bid, or high bid. They therefore gave each potential contractor the dimensions of the proposed runway in the form of the total square footage of the runway. They gave the contractors only 6 hours to come up with the solution for the dimensions. The first to come up with the correct dimensions won the contract. There was never any indication as to the course of action should no one achieve the desired results. They gave the contractors only one hint, namely, that the total square footage was the product of two prime numbers.

My imaginary informant told me that one of the government contracting engineers solved the problem in the 6-hour time period allotted. As serendipity would have it, this engineer just happened to be using his computer with Quattro Pro Optimizer installed. (I am simply relating the imaginary facts, not trying to extol the virtues of Quattro Pro.) This engineer felt like he could solve the problem, but the Optimizer required a Target Value. He therefore set about to devise some sort of equation which would give a Target Value acceptable to the Optimizer program. My imaginary informant says that he took careful notes, in case he should have an occasion to use this method. The author is also passing 
this method along to whoever can use it for whatever engineering purpose that it may happen to fit, if any. The author has attempted the method, but had a bit of trouble with entering formulas with enough parentheses. The Solve button gave incorrect results at first, until the author put in parentheses properly and also used a hand-held calculator to double check results that the Optimizer program gave. When everything worked correctly, the program only took about three seconds to give the proper results.

This is apparently how the engineer did it.

The procurement officials gave the area of the runway as $A=3,945,911$ square feet with the clue that it was a prime product. The engineer created a formula which gave only the smaller of the two dimensions of this area, and devised a formula which gave this smaller dimension with an upper bound to it.

He defined the smaller dimension as $L_{2}=6 S_{2}+\alpha$, where $\alpha=+1$ or -1 .

He then gave the limits of $S_{2}$ as

$$
0<S_{2} \leq \frac{\sqrt{A}+1}{6}
$$

The functions which he used in order to achieve a Target Value, required for the program, are as follows:

$$
\sin \left(\frac{A \pi}{6 S_{2}-1}\right), \sin \left(\frac{A \pi}{6 S_{2}+1}\right)
$$

When the correct value of $S_{2}$ has been reached, the Sine functions go to zero, which is the sought-after Target Value. The Solution Cell in Optimizer is where the Sine functions are entered. The Variable Cell is where the upper bound of $S_{2}$ is entered. The Constraints indicate this upper bound, which is 331, and the fact that $S_{2}$ is an integer, so that Optimizer begins at the upper bound of 331 and works down only using sequential integers, with the final value of $S_{2}=35$, which then yields the value of $L_{2}=211$. The long side or length of the runway is just the area divided by 211 , which yields a length of 18,701 feet or about 3.54 miles. The author has included screen shots of the Quattro Pro spreadsheet and the Optimizer dialogue box for both of the Sine functions. Incidentally, I have checked and both 211 and 18,701 are both prime numbers. See the next two screen shots (Figure 1 and Figure 2) for the details of the calculations. These screen shots are very important for those readers who wish to duplicate the results.

The author also did only one single quick check of the method itself by using prime numbers of 109 and 163 with their product being 17,767. The author calculated the upper bound of $S_{2}=22$, using the engineer's method. There is also an advantage using the Optimizer, as one can change values fairly quickly (remember that the author is not extolling the virtues of Quattro Pro). Using 17,767 as the area and the upper bound of $S_{2}=22$, it was easy to obtain the calculated/iterated value of $S_{2}=18$, from which follows $L_{2}=6(18)+1=109$. Maybe all of this will be of help to some other engineer in somewhat similar circumstances. 


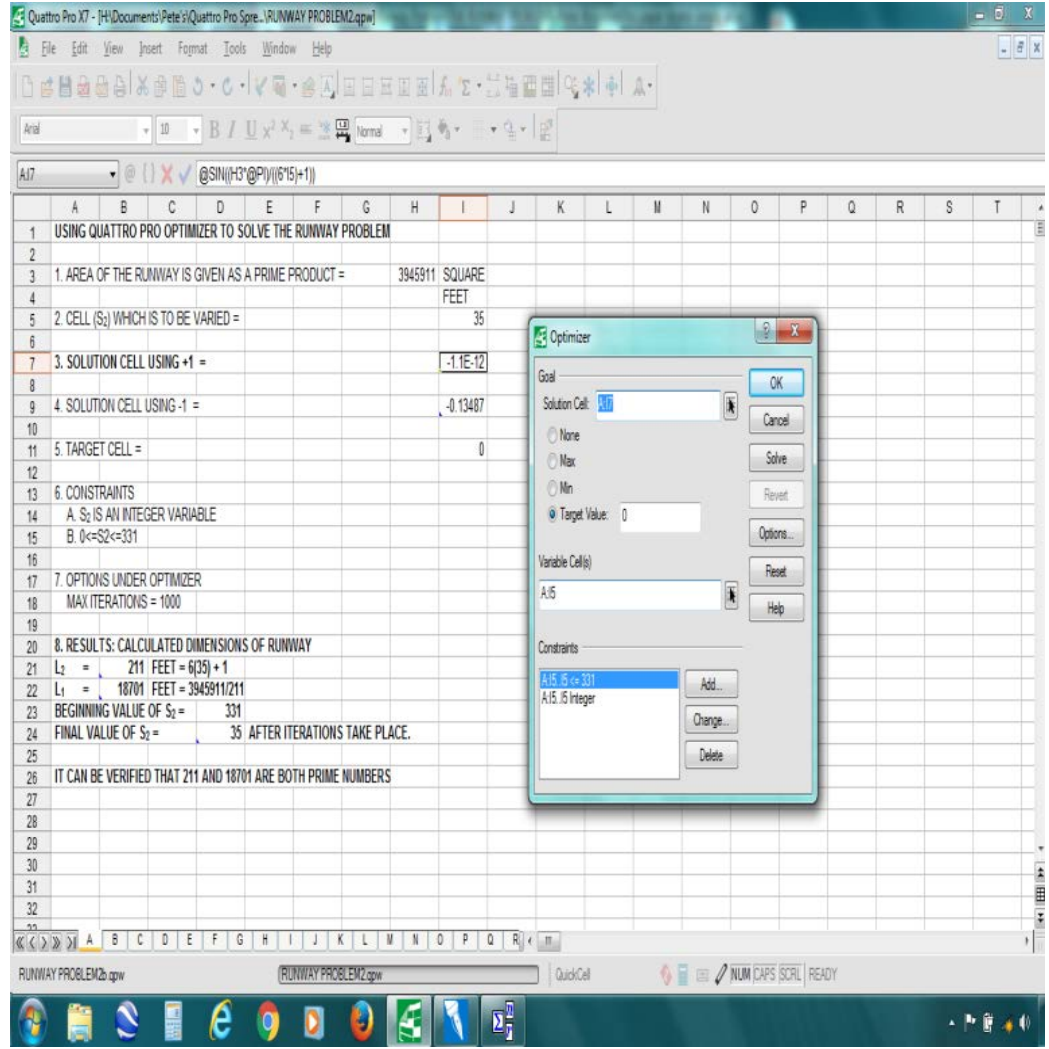

Figure 1. Screen Shot of the Correct Solution of the Runway Problem.

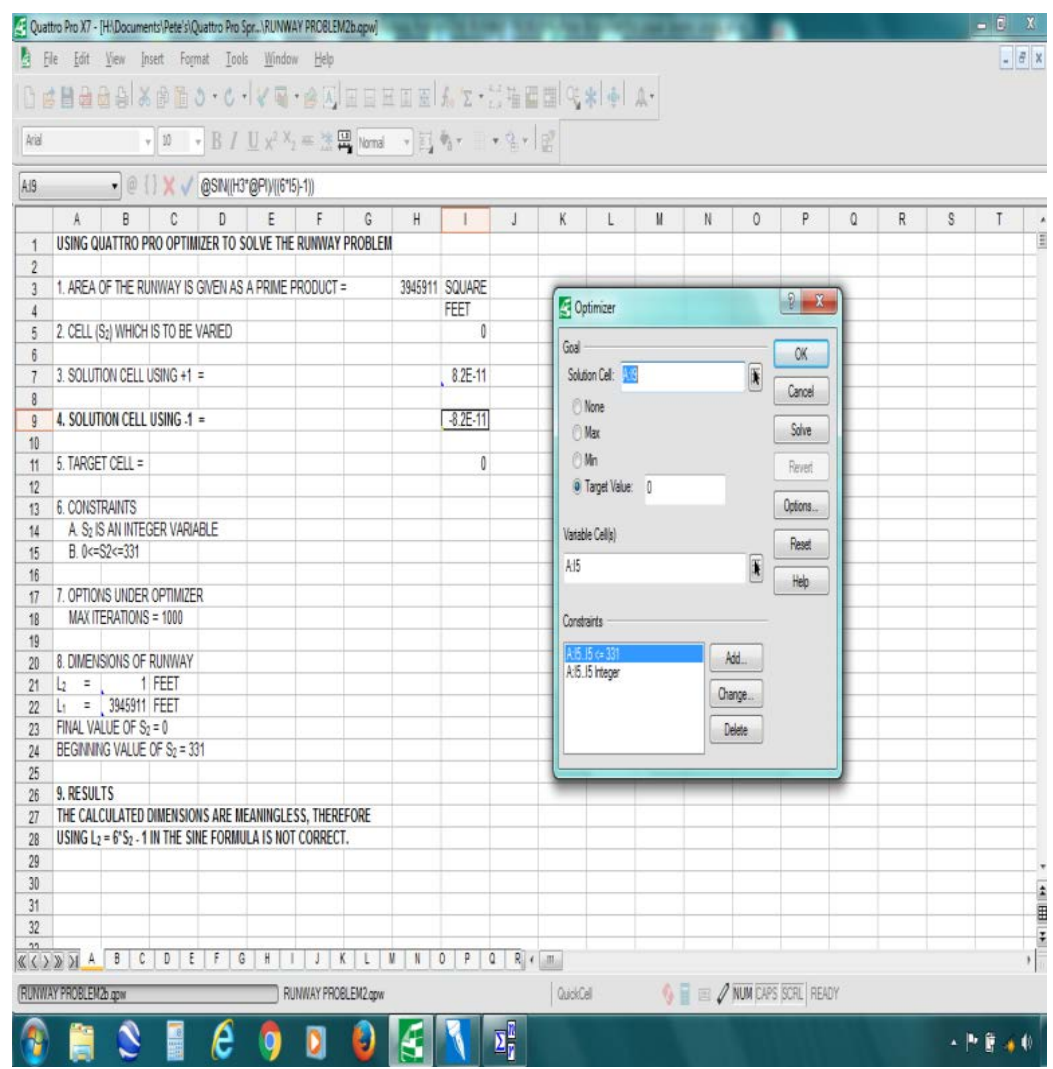

Figure 2. Screen Shot of the Trivial Solution of the Runway Problem. 
Table 3. Eleven Different Integer Solutions for the L's in the Non-Unique Area of 10,000.

\begin{tabular}{cccccccccccc}
\hline$L_{1}$ & 10,000 & 5000 & 2500 & 2000 & 1000 & 500 & 400 & 250 & 200 & 125 & 100 \\
$L_{2}$ & 1 & 2 & 4 & 5 & 10 & 20 & 25 & 40 & 50 & 80 & 100 \\
\hline
\end{tabular}

Using prime products in systems which require security is obvious, as it is complicated to obtain the values of $L_{1}$ or $L_{2}$ which comprise $A$. Prime products may be considered to be unique areas, as there is only set of $L$ 's which satisfy $A$. This is opposed to non-unique areas, such as the number 10,000 which has 11 different solutions for the $L$ integers. See Table 3.

\section{BONUS-How Can You Tell If Your Paper Has Been Plagiarized without Being Plagiarized?}

An anonymous law student thinks he knows how the so called "predator pros" accomplish this in a very smooth and slick fashion. If you desire to plagiarize someone's paper, then the only obstacle is the published paper itself. How do you unpublish it? Well you don't. The trick is called the "red herring" approach, according to this law student. The "predator pro" pulls a red herring out of his hat and theoretically draws it across the path of your paper drawing attention from your paper to a "false source", which he sets up in plain view of everyone, usually with the collusion of other people or even universities, so that it attains the necessary characteristic of unimpeachability. The "predator pro" then declares publicly that he drew his "inspiration" from this "false source", which no one can doubt, and which no one can possibly relate back to your paper. At any rate, once he declares his "inspiration", he then migrates your ideas to this "false source". It then is perceived by everyone, that this "predator pro" could be the originator of your idea. The setting up of the "false source" is the absolute key to the success of this endeavor. One dead giveaway, is that the setup of the "false source" is always dated after your publishing date.

\section{Conclusions}

The overall main difference between this addendum and the main paper of 2006 (neglecting the added analytical and explanatory elucidations) is in attempt at recognizing and presenting better analytical insight (albeit very simplistic in nature) into why the numbers 2 and 3 are not true prime numbers. It is also important to emphasize again that the prime number double helices are a subset of $6 s-1$ and $6 s+1$, involving columns $x=1$ to 6 , with the exception of prime number 7 in row 1 and column 7 . The prime products involving $x=7$ can only be analyzed in the two-dimensional representations previously alluded to.

The second main difference is to warn all scientists and mathematicians of the super-ambitious but low creativity vultures in the mathematical and physical sciences (or in the words of the anonymous law student, "predator pros") who are using more sophisticated psychological methods in attaining their nefarious and undeserved goals and rewards. Sadly, there is a very real possibility that 
some universities worldwide may be involved in sponsoring this behavior.

This paper is important in the larger realm of mathematics, because it presents the novel idea, in opposition to the mainstream of mathematical thought, that mathematics needs to give a better definition of what exactly a prime number really is, and what exactly are the characteristics of prime numbers in reality. What is so pathetic is that when one starts to read articles on prime numbers worldwide, there are still a myriad of ways that people think about the representations of prime numbers - are they random or do they follow a pattern, do they obey some sort of mathematical formula, can they be represented as spirals, helices, triangles, straight lines or whatever? This is disgraceful that there still is no common consensus here in the $21^{\text {st }}$ century. The man-made definition of prime numbers, in the opinion of this author, is simply not good enough! Perhaps in the future, mathematicians will come up with a better definition. Let us hope so, otherwise I fear that the spirit of Fermat will be laughing at all of us!

\section{Acknowledgements}

The author would like to thank the anonymous referee for the feedback that helped to improve this paper.

\section{References}

[1] Bissonnet, P. (2006) Do Prime Numbers Obey a Three-Dimensional Double Helix? Hadronic Journal, 29, 387-400. http://vixra.org/abs/1606.0310

[2] MacDonald, F. (2016) Mathematicians Have Discovered a Strange Pattern Hiding in Prime Numbers. Science Alert. https://www.sciencealert.com/mathematicians-discover-a-strange-pattern-hiding-in -prime-numbers

[3] Double Helix. (2013) A Giant Step for Twin Primes. https://blog.doublehelix.csiro.au/giant-step-twin-primes/

[4] OUPblog (2017) Prime Numbers and How to Find Them. Oxford University Press' Academic Insights for the Thinking World. https://blog.oup.com/2017/05/large-prime-numbers-euclid/ 
Submit or recommend next manuscript to SCIRP and we will provide best service for you:

Accepting pre-submission inquiries through Email, Facebook, LinkedIn, Twitter, etc. A wide selection of journals (inclusive of 9 subjects, more than 200 journals)

Providing 24-hour high-quality service

User-friendly online submission system

Fair and swift peer-review system

Efficient typesetting and proofreading procedure

Display of the result of downloads and visits, as well as the number of cited articles Maximum dissemination of your research work

Submit your manuscript at: http://papersubmission.scirp.org/

Or contact jamp@scirp.org 